\title{
Pre-harvest Sprouting Tolerance of Triticale Genotypes in Brazil
}

\author{
Carlos Henrique dos Santos Fernandes ${ }^{1}$, Klever Márcio Antunes Arruda ${ }^{2}$, Inês Cristina Batista da Fonseca ${ }^{1}$, \\ Claudemir Zucareli ${ }^{1} \&$ Juliana Sawada Buratto ${ }^{2}$ \\ ${ }^{1}$ Agronomy Departament, Universidade Estadual de Londrina, Londrina, Brazil \\ ${ }^{2}$ Plant Breeding Area, Instituto de Desenvolvimento Rural do Paraná, Londrina, Brazil \\ Correspondence: Juliana Sawada Buratto, Plant Breeding Area, Instituto de Desenvolvimento Rural do Paraná, \\ Rodovia Celso Garcia Cid, PR-445, Km 375, 86047-902, Londrina, Brazil. Tel: 55-43-3376-2149. E-mail: \\ jsburatto@idr.pr.gov.br
}

Received: September 8, 2020

Accepted: November 9, $2020 \quad$ Online Published: December 15, 2020

doi:10.5539/jas.v13n1p53

URL: https://doi.org/10.5539/jas.v13n1p53

The research is financed by $C N P q$.

\begin{abstract}
Pre-harvest sprouting (PHS) represents one of the main factors, which causes yield, technological and physiological losses in triticale seeds (X Triticosecale Wittmack). This work aimed to rate the variability and identify potential pre-harvest sprouting tolerant sources in triticale genotypes. Based on that, 32 triticale and three wheat genotypes were sown in 2016, 2017 and 2018 growing seasons, in Londrina-PR, Brazil. After the ears harvesting, these were submitted to simulate raining, for sprouting induction, through nebulization in a greenhouse. After nebulization, ears were sun dried, later hand threshed to determain, grain germination percentage (GERM) and hectoliter weight (HW). Additionally, it was determined grains HW of ears, whom were not submitted to nebulization, as well as the whole meal flour falling number (FN). The experiment design was completely randomized design, with two replications, and the experimental unit was made of 20 ears. The data collected were subjected to analysis of variance (ANOVA) and Scott-Knott test. Frontana, ND 674 and Quartzo are a source of tolerance to PHS in wheat. In triticale genotypes, genetic variability was observed for GERM, FN, and HW prior and after nebulization. The triticale genotypes BRS Netuno, BRS Saturno, TCL 15116, X 092181, Tiguera 1 and Tiguera 8, where tolerant towards PHS.
\end{abstract}

Keywords: falling number, genotype $\times$ environment interaction, hectoliter weight, $\mathrm{X}$ Triticosecale Wittmack

\section{Introduction}

The triticale (X Triticosecale Wittmack), originated from the hybridization of wheat (Triticum) with rye (Secale), aiming to unite favorable traits of both these species. However, triticale cultivars still have deficiencies in some agronomic traits, like susceptibility to certain diseases (fusarium head blight, blast, leaf spots and viruses) (Mergoum et al., 2019).

In 2018, the harvest areas and production of triticale wordwide were 3,809,192 hectares and 12,802,592 tons, respectively (FAO, 2020). Poland, Germany, France, Belarus, China, and Spain are the leading triticale producing countries (FAO, 2020). Triticale can be used for several purposes. Triticale is used in animal feed, it is used especially for direct feeding as green forage or as silage of young or mature plants, moist or dry grains, and hay (Nascimento Junior et al., 2004). In human feeding the triticale grains, its flour has been used to compose mixtures with wheat flour for the manufacture of low fermentation products, such as cookies, pizza dough, meatballs, waffles, and cakes (Watanabe, 2016). Triticale has also aroused interest for biofuel production, and it has presented competitiveness when compared with other winter cereals (McGoverin et al., 2011).

Pre-harvest sprouting (PHS), is a premature grain sprouting in the ear, before the harvested. This problem has been observed over many years in several triticale and wheat growing areas, all over the world. This represents a limiting technological quality factor, to the processing industry and seed production physiological quality (Moś \& Wójtowicz, 2004; Chapman, 2011). In general, dormant grain happens under cool growing conditions, whereas high temperatures during the later stages of grain growth, break embryo dormancy allowing grains to germinate in the ear, granted rain occurs around harvest time. 
Pre-harvest sprouting occurs when the seed interacts with the water, thus the imbibition starts, triggering a slew of physiological processes, like the release of hormones and hydrolytic enzymes. Considered a simplified process, the gibberelic acid hormonal activity on the imbibed seed induce amylases synthesis; hydrolyzed carbohydrates reserves, induce the development of the embryo (Bassoi, 2004).

Many methods where described in scientific literature, assesing pre-harvest sprouting resistance (Nedel et al., 1983a; Humphreys \& Noll, 2002; Gavazza et al., 2012). These methods use the visual assessment of seeds and the falling number, to quantify seed germination percentage and correlate it with seed dormancy.

The PHS genetic variability in triticale is lower in relation to wheat. However, the genetic variability available for triticale, is enough to obtain new cultivars through genetic improvement (Nedel et al., 1983b; Haesaert \& De Baets, 1996; Oettler, 2005; Alaru et al., 2008; Bizova et al., 2011).

The identification of tolerant triticale genotypes, towards PHS, will grant its utilization as parents on breeding programs, thus, contributing to new cultivar development. This work aimed to rate the variability and identify potential pre-harvest sprouting tolerant sources in triticale genotypes.

\section{Method}

\subsection{Material Studied}

The experimental material was composed of 32 triticale genotypes, developed throught triticale genetic improvement programs, by different agencies in Brazil (IAPAR and EMBRAPA) and international institution (CIMMYT). The line ND 674 and the cultivars Quartzo and Frontana were used as controls towards tolerance to pre-harvest sprouting. Frontana cultivar is one of the best wheat genotypes in terms of resistance to pre-harvest germination (Bassoi et al., 2006; Franco et al., 2009; Nörnberg et al., 2015). Quartzo cultivar was classified as moderately tolerant to tolerant to PHS (Nörnberg et al., 2015), and the PHS tolerance of the wheat line ND 674 was reported by Dr. L. Okuyama (personal communication).

\subsection{Site, Weather and Agronomic Management and Data Colletion}

The experiments were carried out in the 2016, 2017 and 2018 growing seasons, in Londrina, state of Paraná, Brazil (latitude $23^{\circ} 22^{\prime} \mathrm{S}$; longitude $51^{\circ} 10^{\prime} \mathrm{W} ; 585 \mathrm{~m}$ altitude). Sowing was carried out in three dates, with intervals of at least one week. Intercalated sowing was used to allow ear harvesting with physiological ripening at the same date. Each field plot was made of 3 lines, $2 \mathrm{~m}$ of length each, spaced $0.3 \mathrm{~m}$. Minimum and maximum temperature $\left({ }^{\circ} \mathrm{C}\right)$, and rainfall $(\mathrm{mm})$ data, were registered and are shown in Figure 1.

Ears were harvested at physiological ripening stage, which is, when they lose their green coloring, but stem nodes are still green. After harvesting, the ears were stored in a protected environment, until grain drying finished (approximately 13\% moisture). After, a fraction of ears were submitted to sprouting induction; the rest were threshed to quantify its hectoliter weight (HW), and after, whole meal flour falling number (FN).

The ears were taken to a greenhouse for nebulization. This method allowed an artificial rain simulation, similar to what occurs in the field. Ears were exposed to nebulization for 42 hours, 68 hours and 42 hours in 2016, 2017 and 2018 growing seasons, respectively. This method has been utilized successfully in the main wheat breeding programs in Brazil, for pre-harvest sprouting resistant genotypes selection (Franco et al., 2009; Okuyama et al., 2017). The experiment design was completely randomized, with two replications, being the experimental unit made of 20 ears.

Grain percentage sprouting (GERM), was determined from ears submitted to nebulization. After nebulization, ears were sun dried and later, hand threshed. GERM was determined based on assessment of two random 50 grain samples on each plot. Germinated grain are those with visible vegetative structure or even visible pericarp breaking.

The FN (also known as Hagberg Number) was determined in samples ( $7 \mathrm{~g}$ ) of ground wheat, which were mixed with $25 \mathrm{~mL}$ of distilled water in a FN tube with a stirrer and shaken to form slurry. This slurry was heated in a boiling water bath at $100{ }^{\circ} \mathrm{C}$ and stirred constantly. The time it took the stirrer to drop through the paste was recorded as the FN value in a Perten FN 1500 (Perten Instruments), according to the 56-81 B method of the American Association of Cereal Chemists (American Association of Cereal Chemists [AACC], 2000).

Hectoliter weight was determined from two sources, from grains which were not submitted to nebulization (HW_s) and from grains originated from ears following nebulization (HW_n). 


\section{Year 2016}
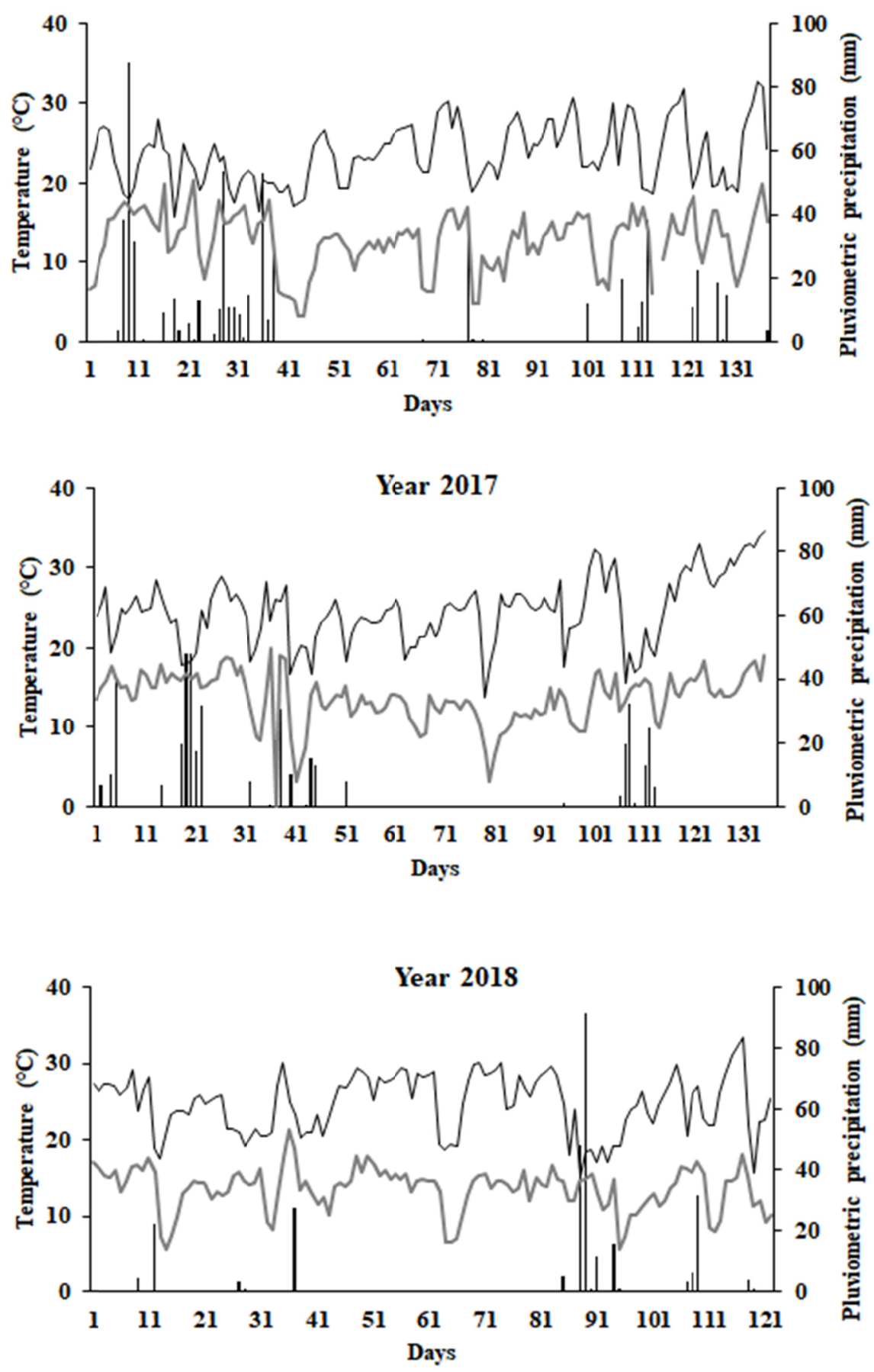

\footnotetext{
Pluviometric precipitation $(\mathrm{mm})-$ Maxim um temperature $\left({ }^{\circ} \mathrm{C}\right)$

Minimum tem perature $\left({ }^{\circ} \mathrm{C}\right)$
}

Figure 1. Rainfall $(\mathrm{mm})$, maximum and minimum temperatures $\left({ }^{\circ} \mathrm{C}\right)$ data during the field experiment course during 2016, 2017 and 2018 (years) growing seasons. Data acquired from the IAPAR Weather Station

\subsection{Statistical Analysis}

The gathered data from sprouting percentage (GERM), falling number (FN), hectoliter weight without nebulization (HW_s) and with nebulization (HW_n) were submitted to individual analysis of variance. Then, residuals variance homogeneity was verified through $\mathrm{F}$ maximum test. Once residuals variance homogeneity was 
verified, joint variance analysis was perfomed, considering genotypes and years effects as fixed. Scott Knott test at $5 \%$ probability was carried out for all traits.

Pearson correlation coefficients were estimated to observe the magnitude and direction between trait pairs. Correlations significance was done through $t$ test with two freedom degrees. Statistical analyses were done with the Genes software aid (Cruz, 2016).

\section{Results and Discussion}

Table 1 demonstrates the variance analyses results for every assessed trait. Significant effect for genotype $(\mathrm{p}<$ 0.05) was observed for all assessed traits. This result indicates the existence if the differences between the evaluated genotypes for hectoliter weight prior and following nebulization, falling number and ear with germinated grains. Considering unfolding within the genotype effect, triticale's significant effect $(p<0.05)$ was observed, indicating performance differences among the genotypes, which allow a selection of superior genotypes. The control effect was not significant $(p>0.05)$, for ear sprouting percentage (GERM), an anticipated fact, as Frontana, Quartzo and ND 674 wheat genotypes, utilized as controls, are rated as tolerants to pre-harvest sprouting. The group significant effect $(\mathrm{p}<0.05)$ reveals that there is a difference among triticale vs wheat groups towards the assessed traits.

Table 1. Joint variance analysis for hectoliter weight prior (HW_s) and following nebulization (HW_n), falling number (FN) and ear with germinated grains (GERM) assessed on the triticales and wheats genotypes. Londrina, State of Paraná, Brazil. 2016, 2017 and 2018 growing seasons

\begin{tabular}{llllll}
\hline \multirow{2}{*}{ Sources of variance } & \multicolumn{5}{c}{ Means Square } \\
\cline { 2 - 6 } & DF & HW_s & HW_n & FN & GERM \\
\hline Genotype $(\mathrm{G})$ & 34 & $17.42^{* *}$ & $30.25^{* *}$ & $35.29^{* *}$ & $1.320^{* *}$ \\
$\quad$ Triticale & 31 & $11.21^{* *}$ & $18.65^{* *}$ & $14.68^{* *}$ & $1.296^{* *}$ \\
Control & 2 & $1.82^{* *}$ & $3.84^{* *}$ & $7.276^{* *}$ & $5.39^{\text {ns }}$ \\
Group & 1 & $241.24^{* *}$ & $442.55^{* *}$ & $730.22^{* *}$ & $4.702^{* *}$ \\
Year & 2 & $17.23^{* *}$ & $597.70^{* *}$ & $198.31^{* *}$ & $6.883^{* *}$ \\
Genotype $\times$ Year & 68 & $7.21^{* *}$ & $7.61^{* *}$ & $5.10^{* *}$ & $323.5^{* *}$ \\
$\quad$ Triticale $\times$ Year & 62 & $6.64^{* *}$ & $6.67^{* *}$ & $3821^{* *}$ & $339.3 * *$ \\
$\quad$ Control $\times$ Year & 4 & $5.52^{* *}$ & $19.54^{* *}$ & $4215^{* *}$ & $10.5^{\text {ns }}$ \\
$\quad$ Group $\times$ Year & 2 & $28.25^{* *}$ & $13.06^{* *}$ & $46.65^{* *}$ & $460.07^{* *}$ \\
Residual & 102 & 0.05 & 0.50 & 18.42 & 19.82 \\
General average & & 77.7 & 70.6 & 143.5 & 18.4 \\
Triticales' Average & & 77.4 & 70.1 & 125.4 & 19.9 \\
Controls' Average & & 81.2 & 75.3 & 336.1 & 2.9 \\
CV $(\%)$ & & 0.29 & 1.00 & 2.99 & 24.2 \\
\hline
\end{tabular}

Note. $\mathrm{HW} \_\mathrm{s}=$ hectoliter weight prior nebulization $\left(\mathrm{kg} \mathrm{hl}^{-1}\right) ; \mathrm{HW} \mathrm{n}=$ hectoliter weight following nebulization $\left(\mathrm{kg} \mathrm{hl}^{-1}\right) ; \overline{\mathrm{FN}}=$ falling number (seconds); GERM $=$ sprouting seed $(\%) .{ }^{\mathrm{ns}}$ not significant by the $\mathrm{F}$ test; ** Significant $(\mathrm{p}<0.01)$ by the $\mathrm{F}$ test; * Significant $(\mathrm{p}<0.05)$ by the $\mathrm{F}$ test.

A significant effect between growing seasons $(\mathrm{p}<0.01)$ for all traits, was detected, indicating that the growing conditions may influence genotypes response. All trials were conducted in the same location, but in different agricultural years. Environmental conditions varied from year to year, this fact can be seen in Figure 1. A significant effect $(p<0.01)$ for the double interactions Genotype $\times$ Year, Control $\times$ Year and Group $\times$ Year was also detected, suggesting differentiated genotype behavior in the different growing seasons (Table 1). Only the interaction Control $\times$ Year for the trait GERM was not significant, since all controls utilized in this study present pre-harvest sprouting tolerance and presented behavior stability on the three years assessed.

Environmental variation coefficients estimates $(\mathrm{CV})$ varied on $0.3 \%$ (hectoliter weight prior nebulization) to $24.8 \%$ (pre-harvest sprouting percentage), which represents good experimental precision for this study according to Gomes (2000).

Among the triticale genotypes, a hectoliter weight variability (HW) was observed. Seed HW prior to nebulization varied from 72 to $81 \mathrm{~kg} \mathrm{~h}^{-1}$ (Table 2). HW express indirectly towards grain quality attributes. 
Therefore, lines and cultivars identification with high values is desirable. Genotypes BRS Netuno, BRS Saturno, IPR Aimoré, PFT 0609, TCL 15014, TCL 15052, TCL 15102 and TCL 0717 presented HW superior to $78 \mathrm{~kg} \mathrm{hl}^{-1}$, in the three assessed years.

The occurrence of harvest raining affects HW negatively, since all genotypes presented HW reduction when submitted to nebulization (Table 2). Due the simulated raining, an average reduction of $9.4 \%$ in the HW (Table 2) was verified. A HW magnitude of reduction due to simulated raining, was distinct among triticale and wheat genotypes (Table 2). Triticale cultivars BRS Saturno, BRS 203 and lines TCL 15052 and TCL 15022 presented the lowest HW reductions, following nebulization. On the other side, genotypes TCL 15102, TCL 11014, ITW 11014 and IPR Aimore were the most effected, since they presented the greatest HW reductions.

Wheat genotypes are set at the greatest tolerance class towards sprouting in all the assessed years (Table 3). Wheat cultivar Frontana, known as a pre-harvest sprouting tolerant source, is much utilized as a parent on breeding programs in Brazil. On the study done by Franco et al. (2009), they reported that dormancy seems to be the most evident mechanism, since the greatest number of dormant seeds detected trough tetrazolium test were associated to the lowest sprouting rates.

Pre-harvest sprouting tolerant cultivars breeding is one of the main challenges to the triticale breeding in the country. The comparative study among the triticales highlighted six promising genotypes: BRS Netuno and BRS Saturno cultivars, TCL 15116 and X 092181 lines and the accesses Tiguera 1 and Tiguera 8. These genotypes had been inserted at the same pre-harvest sprouting control tolerant groups (Frontana, ND 674 e Quartzo) and have presented behavior stability, showing low sprouted grains value on the three years assessed (Table 3). Tolerant genotypes identification permit its use as parents in breeding programs aiming to get future cultivars with great pre-harvest sprouting tolerance levels.

The presence of the Genotype $\times$ Environment $(G \times E)$ interaction makes it difficult to classify genotypes with intermediate HWS resistance. The triticale lines TCL 15022, TCL 15020 and TCLD 1003 were classified as moderately resistant in one season and as moderately susceptible in another season (Table 3).

ITW 11014 and TCLD 0903 were pre-harvest sprouting susceptible, due to the high sprouting values found in the three years of assessment (Table 3). Pre-harvest sprouting is a serious grain quality concern for millers and farmers alike. Sprouting happends as the seed breaks dormancy, and activates enzymes that begin breaking down starch.

Falling numbers is a test that indicates how well the grain's starch has retained its strength. The FN of the triticale genotypes were lower than the wheat genotypes (Table 3). The FN in the triticale genotypes ranged from 62 seconds to FN $>250$ seconds. Climatic conditions influenced this characteristic in different growing season. In 2017, the FN average of triticales was 188 seconds. The average FN in 2016 and 2018 was 99 and 86 seconds, respectively. The higher average FN in 2017 is probably due to absence of rainfall at the end of the grain filling and maturation period (Figure 1).

Falling number trait does not demonstrate correlation with pre-harvest sprouting grains percentage (GERM) on triticale genotypes (Table 4). Such an issue might be explained, by the fact that, triticale genotypes scored the minimum FN the equipment "Falling Number" can register. Triticales' FN cannot be directly compared to wheat, because factors besides $\alpha$-amylase decrease triticale' solution viscosity a lot. What would be considered a low wheat FN number, not necessarily indicates high $\alpha$-amylase activity in triticale. A possible cause is the endogen enzymes action, which does not have $\alpha$-amylase, particularly those which act on non-starch polysaccharides (Randhawa et al., 2015).

A high correlation was detected, although negative, between pre-harvest sprouting grains percentage (GERM) and nebulized grains hectoliter weight (HW_n) on the three assessed years (Table 4). Therefore, grain HW quantifying following simulated rain may be used to the aid screening pre-harvest sprouting tolerant triticale genotypes. 
Table 2. Hectoliter weight average $\left(\mathrm{kg} \mathrm{hl}^{-1}\right)$ on triticale and wheat seeds assessed prior (HW_s) and following nebulization (HW_n). 2016, 2017 and 2018 growing seasons

\begin{tabular}{|c|c|c|c|c|c|c|}
\hline \multirow{2}{*}{ Genotype } & \multicolumn{3}{|c|}{ HW_s $\left(\mathrm{kg} \mathrm{hl}^{-1}\right)$} & \multicolumn{3}{|c|}{ HW_n $\left(\mathrm{kg} \mathrm{hl}^{-1}\right)$} \\
\hline & 2016 & 2017 & 2018 & 2016 & 2017 & 2018 \\
\hline BRS 203 & $79 \mathrm{f}$ & $76 \mathrm{~h}$ & $76 \mathrm{j}$ & $72 \mathrm{c}$ & $69 \mathrm{c}$ & $74 \mathrm{e}$ \\
\hline BRS Harmonia & $76 \mathrm{i}$ & $76 \mathrm{~h}$ & $79 \mathrm{f}$ & $66 \mathrm{~g}$ & $70 \mathrm{c}$ & $72 \mathrm{f}$ \\
\hline BRS Netuno & $78 \mathrm{f}$ & $78 \mathrm{f}$ & $79 \mathrm{f}$ & $72 \mathrm{c}$ & $69 \mathrm{~d}$ & $75 \mathrm{~d}$ \\
\hline BRS Saturno & $79 \mathrm{f}$ & $79 \mathrm{~d}$ & $79 \mathrm{~g}$ & $74 \mathrm{~b}$ & $74 \mathrm{a}$ & $74 \mathrm{e}$ \\
\hline IPR 111 & 731 & $77 \mathrm{~g}$ & $75 \mathrm{k}$ & $66 \mathrm{~g}$ & $67 \mathrm{e}$ & $68 \mathrm{~h}$ \\
\hline IPR Aimoré & $79 \mathrm{e}$ & $78 \mathrm{e}$ & $79 \mathrm{~g}$ & $71 \mathrm{~d}$ & $65 \mathrm{f}$ & $73 \mathrm{f}$ \\
\hline ITW 11014 & $74 \mathrm{k}$ & $76 \mathrm{~h}$ & $79 \mathrm{e}$ & $65 \mathrm{~g}$ & $65 \mathrm{f}$ & $72 \mathrm{f}$ \\
\hline PFT 0609 & 79 e & $80 \mathrm{a}$ & $78 \mathrm{~g}$ & $72 \mathrm{c}$ & $72 \mathrm{~b}$ & $75 \mathrm{~d}$ \\
\hline TCL 13003 & $81 \mathrm{c}$ & $80 \mathrm{a}$ & $77 \mathrm{i}$ & $71 \mathrm{~d}$ & $68 \mathrm{~d}$ & $69 \mathrm{~h}$ \\
\hline TCL 15008 & $76 \mathrm{i}$ & $77 \mathrm{f}$ & $78 \mathrm{~g}$ & $71 \mathrm{~d}$ & $67 \mathrm{e}$ & $75 \mathrm{~d}$ \\
\hline TCL 15014 & $78 \mathrm{f}$ & $78 \mathrm{f}$ & $78 \mathrm{~g}$ & $70 \mathrm{~d}$ & $69 \mathrm{c}$ & $71 \mathrm{~g}$ \\
\hline TCL 15019 & $78 \mathrm{f}$ & $77 \mathrm{~g}$ & $77 \mathrm{i}$ & $69 \mathrm{e}$ & $67 \mathrm{e}$ & $73 \mathrm{e}$ \\
\hline TCL 15020 & $77 \mathrm{~g}$ & $78 \mathrm{e}$ & $77 \mathrm{i}$ & $71 \mathrm{~d}$ & $69 \mathrm{~d}$ & $74 \mathrm{e}$ \\
\hline TCL 15022 & $75 \mathrm{k}$ & $75 \mathrm{j}$ & $78 \mathrm{~h}$ & $69 \mathrm{e}$ & $67 \mathrm{e}$ & $75 \mathrm{~d}$ \\
\hline TCL 15052 & $79 \mathrm{~d}$ & $78 \mathrm{e}$ & $80 \mathrm{~d}$ & $73 \mathrm{~b}$ & $71 \mathrm{c}$ & $77 \mathrm{c}$ \\
\hline TCL 15063 & $82 \mathrm{~b}$ & $77 \mathrm{f}$ & $76 \mathrm{i}$ & $70 \mathrm{~d}$ & $70 \mathrm{c}$ & $75 \mathrm{~d}$ \\
\hline TCL 15102 & $79 \mathrm{f}$ & $78 \mathrm{e}$ & $80 \mathrm{e}$ & $68 \mathrm{f}$ & $66 \mathrm{f}$ & $72 \mathrm{f}$ \\
\hline TCL 15104 & $78 \mathrm{~g}$ & $78 \mathrm{e}$ & $77 \mathrm{i}$ & $68 \mathrm{f}$ & $64 \mathrm{f}$ & $73 \mathrm{e}$ \\
\hline TCL 15116 & $80 \mathrm{~d}$ & $78 \mathrm{e}$ & $77 \mathrm{i}$ & $68 \mathrm{f}$ & $72 b$ & $74 \mathrm{e}$ \\
\hline TCLD 0717 & $80 \mathrm{~d}$ & $79 \mathrm{c}$ & $78 \mathrm{~g}$ & $69 \mathrm{e}$ & $68 \mathrm{~d}$ & $74 \mathrm{e}$ \\
\hline TCLD 0903 & $76 \mathrm{j}$ & $79 \mathrm{c}$ & $77 \mathrm{i}$ & $71 \mathrm{~d}$ & $67 \mathrm{e}$ & $72 \mathrm{f}$ \\
\hline TCLD 1003 & $80 \mathrm{~d}$ & $74 \mathrm{k}$ & 741 & $68 \mathrm{f}$ & $68 \mathrm{~d}$ & $72 \mathrm{f}$ \\
\hline Tiguera 1 & $76 \mathrm{j}$ & $75 \mathrm{j}$ & $76 \mathrm{j}$ & $67 \mathrm{f}$ & $67 \mathrm{e}$ & $75 \mathrm{~d}$ \\
\hline Tiguera 5 & $79 \mathrm{e}$ & $76 \mathrm{~h}$ & $78 \mathrm{~h}$ & $72 \mathrm{c}$ & $66 \mathrm{e}$ & $75 \mathrm{~d}$ \\
\hline Tiguera 6 & $76 \mathrm{j}$ & 731 & $78 \mathrm{~h}$ & $68 \mathrm{f}$ & $67 \mathrm{e}$ & $76 \mathrm{~d}$ \\
\hline Tiguera 8 & $80 \mathrm{~d}$ & $75 \mathrm{j}$ & $79 \mathrm{e}$ & $71 \mathrm{~d}$ & $68 \mathrm{~d}$ & $73 \mathrm{e}$ \\
\hline TLD 1204 & $77 \mathrm{~h}$ & $79 \mathrm{~d}$ & $79 \mathrm{~g}$ & $69 \mathrm{e}$ & $67 \mathrm{e}$ & $73 \mathrm{f}$ \\
\hline TPOLO 0611 & $72 \mathrm{~m}$ & $75 \mathrm{j}$ & $76 \mathrm{j}$ & $64 \mathrm{~h}$ & $63 \mathrm{~g}$ & $73 \mathrm{e}$ \\
\hline TPOLO 0628 & 731 & $77 \mathrm{~g}$ & $78 \mathrm{~h}$ & $68 \mathrm{f}$ & $69 \mathrm{~d}$ & $71 \mathrm{~g}$ \\
\hline TPOLO 61 & $76 \mathrm{j}$ & $78 \mathrm{f}$ & $78 \mathrm{~h}$ & $65 \mathrm{~h}$ & $66 \mathrm{e}$ & $71 \mathrm{~g}$ \\
\hline TPOLO 66 & $81 \mathrm{~b}$ & $77 \mathrm{~g}$ & $78 \mathrm{~h}$ & $73 \mathrm{c}$ & $67 \mathrm{e}$ & $75 \mathrm{~d}$ \\
\hline X 092181 & $72 \mathrm{~m}$ & $75 \mathrm{i}$ & $80 \mathrm{~d}$ & $66 \mathrm{~g}$ & $67 \mathrm{e}$ & $76 \mathrm{~d}$ \\
\hline Frontana (WTC*) & $81 \mathrm{c}$ & $80 \mathrm{~b}$ & $82 \mathrm{c}$ & $76 \mathrm{a}$ & $72 b$ & $77 \mathrm{c}$ \\
\hline ND 674 (WTC*) & $83 \mathrm{a}$ & $76 \mathrm{~h}$ & $84 \mathrm{a}$ & $76 \mathrm{a}$ & $67 \mathrm{e}$ & $81 \mathrm{a}$ \\
\hline Quartzo (WTC*) & $84 \mathrm{a}$ & $79 \mathrm{c}$ & $83 \mathrm{~b}$ & $75 \mathrm{~b}$ & $75 \mathrm{a}$ & $79 \mathrm{~b}$ \\
\hline
\end{tabular}

Note. Averages followed by the same letter in column are not different among itself according to Scott Knott test at $5 \%$. ${ }^{*}$ WTC $=$ Wheat Tolerance Control. 
Table 3. Falling Number (FN) and pre-harvest sprouting (GERM) assessed in 32 and 3, triticale and wheat genotypes, respectively. Londrina, PR, Brazil. 2016, 2017 and 2018 growing seasons

\begin{tabular}{|c|c|c|c|c|c|c|}
\hline \multirow{2}{*}{ Genotype } & \multicolumn{3}{|c|}{ FN (s) } & \multicolumn{3}{|c|}{ GERM (\%) } \\
\hline & 2016 & 2017 & 2018 & 2016 & 2017 & 2018 \\
\hline BRS 203 & $253 \mathrm{c}$ & $284 \mathrm{f}$ & $70 \mathrm{j}$ & $8 \mathrm{c}$ & $6 \mathrm{f}$ & $15.5 \mathrm{c}$ \\
\hline BRS Harmonia & 621 & $89 \mathrm{q}$ & $66 \mathrm{j}$ & $46 \mathrm{a}$ & $44 \mathrm{c}$ & $31 \mathrm{a}$ \\
\hline BRS Netuno & 621 & $239 \mathrm{~h}$ & $69 \mathrm{j}$ & $3 \mathrm{~d}$ & $7 \mathrm{f}$ & $6 \mathrm{~d}$ \\
\hline BRS Saturno & $79 \mathrm{k}$ & $301 \mathrm{~d}$ & $210 \mathrm{e}$ & $2 \mathrm{~d}$ & $14 \mathrm{f}$ & $4.5 \mathrm{~d}$ \\
\hline IPR 111 & 631 & 114 o & $62 \mathrm{k}$ & $2 \mathrm{~d}$ & $45 \mathrm{c}$ & $30.5 \mathrm{a}$ \\
\hline IPR Aimoré & 641 & 118 o & $62 \mathrm{k}$ & $12 \mathrm{c}$ & $86 \mathrm{a}$ & $37.5 \mathrm{a}$ \\
\hline ITW 11014 & $124 \mathrm{~h}$ & $163 \mathrm{~m}$ & $72 \mathrm{j}$ & $36 \mathrm{a}$ & $88 \mathrm{a}$ & $32.5 \mathrm{a}$ \\
\hline PFT 0609 & $100 \mathrm{j}$ & 124 o & $62 \mathrm{k}$ & $16 \mathrm{c}$ & $21 \mathrm{e}$ & $14.5 \mathrm{c}$ \\
\hline TCL 13003 & $192 \mathrm{~d}$ & $125 \mathrm{i}$ & $62 \mathrm{k}$ & $13 \mathrm{c}$ & $78 \mathrm{a}$ & $36.5 \mathrm{a}$ \\
\hline TCL 15008 & $156 \mathrm{f}$ & $211 \mathrm{j}$ & $68 \mathrm{j}$ & $3 d$ & $35 \mathrm{~d}$ & $11 \mathrm{c}$ \\
\hline TCL 15014 & 621 & $157 \mathrm{~m}$ & $63 \mathrm{k}$ & $1 \mathrm{~d}$ & $2 \mathrm{f}$ & $12 \mathrm{c}$ \\
\hline TCL 15019 & 621 & $100 \mathrm{p}$ & $62 \mathrm{k}$ & $6 \mathrm{~d}$ & $29 \mathrm{~d}$ & $5.5 \mathrm{~d}$ \\
\hline TCL 15020 & $96 \mathrm{j}$ & 1811 & $65 \mathrm{k}$ & $8 \mathrm{c}$ & $24 \mathrm{e}$ & $20.5 \mathrm{~b}$ \\
\hline TCL 15022 & 621 & $103 \mathrm{p}$ & $62 \mathrm{k}$ & $10 \mathrm{c}$ & $21 \mathrm{e}$ & $9.5 \mathrm{c}$ \\
\hline TCL 15052 & 641 & $245 \mathrm{~h}$ & $62 \mathrm{k}$ & $6 \mathrm{~d}$ & $26 \mathrm{~d}$ & $24 \mathrm{~b}$ \\
\hline TCL 15063 & $134 \mathrm{~g}$ & $267 \mathrm{~g}$ & $108 \mathrm{~h}$ & $1 \mathrm{~d}$ & $42 \mathrm{c}$ & $9 \mathrm{~d}$ \\
\hline TCL 15102 & 621 & $141 \mathrm{n}$ & $62 \mathrm{k}$ & $7 \mathrm{~d}$ & $60 \mathrm{~b}$ & $6.5 \mathrm{~d}$ \\
\hline TCL 15104 & 681 & $139 n$ & $69 \mathrm{j}$ & $12 \mathrm{c}$ & $58 \mathrm{~b}$ & $6.5 \mathrm{~d}$ \\
\hline TCL 15116 & $111 \mathrm{i}$ & $137 n$ & $62 \mathrm{k}$ & $4 \mathrm{~d}$ & $4 \mathrm{f}$ & $3.5 \mathrm{~d}$ \\
\hline TCLD 0717 & $72 \mathrm{k}$ & 1841 & $62 \mathrm{k}$ & $4 \mathrm{~d}$ & $16 \mathrm{e}$ & $6.5 \mathrm{~d}$ \\
\hline TCLD 0903 & 621 & $230 \mathrm{i}$ & $62 \mathrm{k}$ & $38 \mathrm{a}$ & $86 \mathrm{a}$ & $40.5 \mathrm{a}$ \\
\hline TCLD 1003 & 621 & $159 \mathrm{~m}$ & $62 \mathrm{k}$ & $18 \mathrm{c}$ & $19 \mathrm{e}$ & $15 \mathrm{c}$ \\
\hline Tiguera 1 & $183 \mathrm{e}$ & $295 \mathrm{e}$ & $248 \mathrm{c}$ & $2 \mathrm{~d}$ & $2 \mathrm{f}$ & $6.5 \mathrm{~d}$ \\
\hline Tiguera 5 & $73 \mathrm{k}$ & $292 \mathrm{e}$ & $71 \mathrm{j}$ & $12 \mathrm{c}$ & $5 \mathrm{f}$ & $1.5 \mathrm{~d}$ \\
\hline Tiguera 6 & $132 \mathrm{~g}$ & $192 \mathrm{k}$ & $63 \mathrm{k}$ & $2 d$ & $20 \mathrm{e}$ & $6 \mathrm{~d}$ \\
\hline Tiguera 8 & $115 \mathrm{i}$ & $85 \mathrm{q}$ & $70 \mathrm{j}$ & $2 \mathrm{~d}$ & $5 \mathrm{f}$ & $8 \mathrm{~d}$ \\
\hline TLD 1204 & 621 & $290 \mathrm{e}$ & $107 \mathrm{~h}$ & $26 \mathrm{~b}$ & $42 \mathrm{c}$ & $37.5 \mathrm{a}$ \\
\hline TPOLO 0611 & $89 \mathrm{j}$ & $281 \mathrm{f}$ & $94 \mathrm{i}$ & $23 \mathrm{~b}$ & $60 \mathrm{~b}$ & $8.5 \mathrm{~d}$ \\
\hline TPOLO 0628 & 631 & $77 \mathrm{r}$ & $62 \mathrm{k}$ & $38 \mathrm{a}$ & $27 \mathrm{~d}$ & $17.5 \mathrm{c}$ \\
\hline TPOLO 61 & 671 & $238 \mathrm{~h}$ & $148 \mathrm{~g}$ & $18 \mathrm{c}$ & $32 \mathrm{~d}$ & $31 \mathrm{a}$ \\
\hline TPOLO 66 & 671 & $137 n$ & $62 \mathrm{k}$ & $4 \mathrm{~d}$ & $22 \mathrm{e}$ & $11.5 \mathrm{c}$ \\
\hline X 092181 & $256 \mathrm{c}$ & $317 \mathrm{c}$ & $237 \mathrm{~d}$ & $1 \mathrm{~d}$ & $6 \mathrm{f}$ & $1.5 \mathrm{~d}$ \\
\hline Frontana (WTC*) & $365 \mathrm{~b}$ & $354 \mathrm{a}$ & $176 \mathrm{f}$ & $1 \mathrm{~d}$ & $2 \mathrm{f}$ & $5 \mathrm{~d}$ \\
\hline ND 674 (WTC*) & $438 \mathrm{a}$ & $307 d$ & $287 \mathrm{~b}$ & $1 \mathrm{~d}$ & $6 \mathrm{f}$ & $0 \mathrm{~d}$ \\
\hline Quartzo (WTC*) & $443 \mathrm{a}$ & $344 \mathrm{~b}$ & $313 \mathrm{a}$ & $3 \mathrm{~d}$ & $7 \mathrm{f}$ & $2.5 \mathrm{~d}$ \\
\hline
\end{tabular}

Note. Averages followed by the same letter in column are not different among itself according to Scott Knott test at $5 \%$. WTC $=$ Wheat Tolerance Control. 
Table 4. Pearson correlation among traits of pre-harvest sprouting in wheat and triticale genotypes in the years 2016, 2017 and 2018

\begin{tabular}{|c|c|c|c|c|}
\hline Trait & HW_s & HW_n & $\mathrm{FN}$ & GERM \\
\hline \multicolumn{5}{|c|}{ Year 2016} \\
\hline HW_s & 1 & $0.74 * *$ & $0.40 *$ & $-0.43 *$ \\
\hline HW_n & & 1 & $0.47 * *$ & $-0.41 *$ \\
\hline $\mathrm{FN}$ & & & 1 & ns \\
\hline GERM & & & & 1 \\
\hline \multicolumn{5}{|c|}{ Year 2017} \\
\hline HW_s & 1 & $0.44 * *$ & ns & ns \\
\hline HW_n & & 1 & ns & $-0.50 * *$ \\
\hline $\mathrm{FN}$ & & & 1 & ns \\
\hline GERM & & & & 1 \\
\hline \multicolumn{5}{|c|}{ Year 2018} \\
\hline HW_s & 1 & $0.65 * *$ & $0.53 * *$ & ns \\
\hline HW_n & & 1 & $0.58 * *$ & $-0.54 * *$ \\
\hline $\mathrm{FN}$ & & & 1 & ns \\
\hline GERM & & & & 1 \\
\hline
\end{tabular}

Note. ${ }^{* *}, *$ Significant at 1 e 5\%, respectively. ns: not significant. T test with $\mathrm{n}-2$ freedom degree.

HW_s: hectoliter weight without nebulization; HW_n: hectoliter weight with nebulization; FN: falling number; GERM: germination percentage.

\section{Conclusion}

There is a variability for pre-harvest sprouting tolerance among triticale genotypes assessed in this study. Triticale genotypes BRS Netuno, BRS Saturno, TCL 15116, X 092181, Tiguera 1 and Tiguera 8, standout by showing comparable tolerance, to the one observed in pre-harvest sprouting tolerant wheat acknowledged cultivars. The most tolerant genotypes could be used as parents in the future breeding programs, towards developing varieties with pre-harvest sprouting tolerance.

\section{Acknowledgements}

The authors wish to acknowledge the CNPq and IDR-Paraná by providing financial resources, sctructural and scholarship.

\section{References}

AACC (American Association of Cereal Chemists). (2000) Approved Methods of the American Association of Cereal Chemists (10th ed.). AACC.

Alaru, M., Laur, Ü., \& Lauringson, E. (2008). Pre-harvest sprouting tolerance of different winter triticale cultivars in the Baltic Sea area. Acta Agricultura eScandinavica Section B: Soil and Plant Science, 58(1), 11-16. https://doi.org/10.1080/09064710601079575

Bassoi, M. C. (2004) Aspectos gerais da germinação pré-colheita e seu controle genético. In G. R. Cunha \& J. L. Pires (Eds.), Germinação pré-colheita em trigo (pp. 21-136). Passo Fundo, RS: Embrapa Trigo.

Bassoi, M. C., Flintham, J., \& Riede, C. R. (2006). Analysis of pre harvest sprouting in three Brazilian wheat populations. Pesquisa Agropecuária Brasileira, 41(4), 583-590. https://doi.org/10.1590/S0100-204X20060 00400006

Bizova, I., Hromadko, M., \& Svec, K. (2011). Testing of pre-harvest sprouting of wheat and triticale at the breeding station Uhretice. Selgen Corp. Czech Republic.

Chapman, B. (2011). Economic impact of pre-harvest sprouting, seed dormancy and germination from a famer perspective. Proc 12th IntSymp Pre-harvest sprouting in cereals (p. 11). Alberta, Canada.

Cruz, C. D. (2016). Genes Software-extended and integrated with the R, Matlab and Selegen. Acta Scientiarum. Agronomy, 38(4), 547-552. https://doi.org/10.4025/actasciagron.v38i4.32629

FAO (Food and Agriculture organization of the United Nations). (2020). FAOSTAT. Retrieved from http://www.fao.org/faostat/en/\#data/QC 
Flintham, J. E. (2000). Different genetic components control coat-imposed and embryo-imposed dormancy in wheat. Seed Science Research, 10(1), 43-50. https://doi.org/10.1017/S0960258500000052

Franco, F. D. A., Pinto, R. J. B., Scapim, C. A., Schuster, I., Predebon, C. T., \& Marchioro, V. S. (2009). Tolerância à germinação na espiga em cultivares de trigo colhido na maturação fisiológica. Ciência Rural, 39(9), 2396-2401. https://doi.org/10.1590/S0103-84782009005000212

Gavazza, M. I. A., Bassoi, M. C., Carvalho, T. C., Bespalhok Filho, J. C., \& Panobianco, M. (2012). Methods for assessment of pre-harvest sprouting in wheat cultivars. Pesquisa Agropecuária Brasileira, 47(7), 928-933. https://doi.org/10.1590/S0100-204X2012000700008

Gomes, F. P. (2000). Curso de estatistica experimental (14th ed.). Piracicaba: Esalq.

Haesaert, G., \& De Baets, A. E. G. (1996). Pre-harvest sprouting resistance in triticale: Preliminary result. In H. Guedes-Pinto, N. Darvey, \& V. P. Carnide (Eds.), Triticale: Today and Tomorrow (Vol. 5, pp. 615-622). Developments in Plant Breeding, Springer: Dordrecht. https://doi.org/10.1007/978-94-009-0329-6_79

Humphreys, D. G., \& Noll, J. (2002). Methods for characterization of preharvest sprouting resistance in a wheat breeding program. Euphytica, 126, 61-65. https://doi.org/10.1023/A:1019671622356

McGoverin, C. M., Snyders. F., Muller, N., Botes, W., Fox, G., \& Manley, M. (2011) A review of triticale uses and the effect of growth environment on grain quality. Journal of the Science of Food and Agriculture, 91, 1155-1165. https://doi.org/10.1002/jsfa.4338

Mergoum, M., Sapkota, S., Eldoliefy, A. E. A., Naraghi, S. M., Pirseyedi, S., Alamri, M. S., \& AbuHammad, W. (2019). Triticale (X Triticosecale Wittmack) breeding. In J. Al-Khayri, S. Jain, \& D. Johnson (Eds.), Advances in Plant Breeding Strategies: Cereals (pp. 405-451). Springer: Cham. https://doi.org/10.1007/ 978-3-030-23108-8_11

Moś, M., \& Wójtowicz, T. (2004). The effect of seed sprouting damage on field emergence and yield of spring triticale. Journal of Central European Agriculture, 5(4), 251-258.

Nascimento Junior, A., Baier, A. C., Teixeira, M. C. C., \& Wiethölter, S. (2004). Triticale in Brazil. In M. Mergoum \& H. G. MacPherson (Eds.), Triticale improvement and production (1st ed., pp. 93-98). FAO, Roma.

Nedel, J. L., Baier, A. C., \& Ignaczak, J. C. (1983a). Comparação de testes para avaliar a germinação na espiga em triticale. Revista Brasileira Sementes, 5(1), 75-80. https://doi.org/10.17801/0101-3122/rbs.v5n1p75-80

Nedel, J. L., Baier, A. C., Linhares, A. G., \& Dias, J. C. A. (1983b). Avaliação de linhagens de triticale quanto à germinação na espiga. Revista Brasileira Sementes, 5, 69-74. https://doi.org/10.17801/0101-3122/rbs. v5n2p69-74

Nörnberg, R., Silva, J. A. G. D., Luche, H. D. S., Tessmann, E. W., Kavalco, S. A. F., Zimmer, C. M., \& Oliveira, A. C. D. (2015). Tolerance to preharvest sprouting and yield of wheat genotypes from different breeding programs. Pesquisa Agropecuária Brasileira, 50(8), 698-706. https://doi.org/10.1590/S0100-204X2015000 800008

Oettler, G. (2005). The fortune of a botanical curiosity_-Triticale: Past, present and future. The Journal of Agricultural Science, 143(5), 329-346. https://doi.org/10.1017/S0021859605005290

Okuyama, L. A., Fonseca Júnior, N. S., Caramori, P. H., \& Kohli, M. M. (2017). Preharvest sprouting assessment in wheat genotypes influenced by temperature and degree days. Experimental Agriculture, 54(4), 483-490. https://doi.org/10.1017/S0014479717000114

Randhawa, H. S., Bona, L., \& Graf, R. J. (2015). Triticale breeding-Progress and prospect. In F. Eudes (Ed.), Triticale (pp.15-32). New York: Springer. https://doi.org/10.1007/978-3-319-22551-7_2

Watanabe, E. (2016). Caracterização fisico-química e reológica de triticale (X Triticosecale Wittmack) visando à aplicação em biscoito tipo cookie. Londrina: Brazil.

\section{Copyrights}

Copyright for this article is retained by the author(s), with first publication rights granted to the journal.

This is an open-access article distributed under the terms and conditions of the Creative Commons Attribution license (http://creativecommons.org/licenses/by/4.0/). 